\title{
Cytological and Morphological Studies in the Genus Sorghum
}

\author{
M. L. Magoon ${ }^{1}$, P. L. Manchanda and M. S. Ramanna \\ Division of Botany \\ Indian Agricultural Research Institute, New Delhi-12, India \\ Received March 8, 1963
}

\section{Introduction}

The ancestral relationship between different taxa is a fascinating problem in any group of plants; this is particularly so in a complex group like the Sorghum, though unravelling process has been one of considerable difficulty. That the conventional approach of studying chromosome pairing at metaphase I and of analyzing the mitotic chromosome morphology has several limitations, has already been amply demonstrated (Magoon and Shambulingappa 1960, Magoon et al. 1961, Magoon and Ramanna 1961 a and b). Information of far greater precision can be obtained by the study of pachytene stage in the meiosis of taxa within this complex and in hybrids between them. This is apparent when we consider that in the mitotic metaphase one is dealing with chromosome lengths varying only in a few micra (e.g., about $3 \mu$ in $S$. ankolib, Magoon and Shambulingappa 1960) while in the pachytene morphology the magnitude of variation is very high (about $50 \mu$ in S. ankolib, Magoon and Shambulingappa, 1960) resulting in much less error in classifying the chromosomes on the basis of their size. Several karyomorphological features not observable in the somatic chromosomes such as the chromomeric pattern, extent of eu- and hetero-chromatic segments, nucleolus organizing regions of the chromosomes and in some cases special karyotypic landmarks like the knobs etc. are also available for detailed study.

Further, structural differences that are not normally manifest in the study of metaphase pairing, become very striking at the pachytene stage. Consequently information that would otherwise be not available can be obtained in a study of the early stages of meiosis and if considered critically can be of immense value in deriving homologies and tracing ancestral relationships. While not all plant material are amenable to this approach, valuable information with regard to several Sorghum species has already been obtained (Magoon 1961). In order to get a complete picture of the interrelationship amongst the different species in this highly variable group, it is necessary to extend this analysis to as many species as are available and in the present

1 Present address: Director, Main Tuber Crops Research Station, Sreekariyam, Trivandrum-II, India. 
paper some hitherto unanalyzed species and some of their hybrid combination are considered.

\section{Materials and methods} study.

The following species and species hybrids were used in the present

1. S. technicum, 2. S. verticilliflorum, 3. S. nitens, 4. S. virgatum,

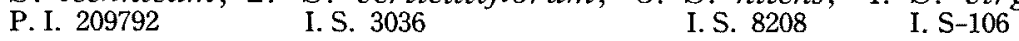

5. S. caudatum, 6. S. vulgare, 7. S. melaleucum, 8. S. caffrorum, $\begin{array}{llll}\text { I.S. } 3144 & \text { I.S. } 1517 & \text { I.S. } 3104 & \text { I.S. } 3137\end{array}$

9. S. subglabrascens, 10. S. mellitum, 11. S. bicolor, 12. S. coriaceum,

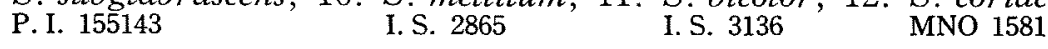

13. S. halepense, 14. S. technicum $\times$ S. virgatum, 15. S. verticilliflorum I. S. 3304

$\times S$. subglabrascens 16. S. nitens $\times S$. caffrorum, 17. S. vulgare

$\times S$. verticilliflorum, $18 . S$. melaleucum $\times S$. vulgare, 19. S. caudatum

$\times S$. nitens.

All the crosses were effected under controlled conditions. Parents and their hybrids grown in the field as well as those grown in the greenhouse were used for the study of morphology and microsporogenesis. For meiotic studies the propiono-carmine smears (Swaminathan et al. 1954 and Magoon et al. 1958) were used. Since the pachytene chromosomes in the material under study were found to be somewhat similar to those of tomato, straight line drawings were used for measuring length etc. as suggested by Barton (1950), Gottschalk (1954) and Magoon et al. (1961). For the sake of convenience, in comparing the chromosome sizes "Relative length" was used which represents the ratio in percentage of the length of the individual chromosome to that of the longest in the complement (Huziwara 1956).

\section{Results}

Chromosome morphology and pairing behaviour

\section{a) Species}

Based on average values obtained by critical measurements in several nuclei at mid pachytene where all chromosomes could be traced from one to other, the ten pachytene bivalents of each species have been identified (Figs. 1 to 3 ) and arranged in the decreasing order of their length. Hence chromosome I was the longest and chromosome $\mathrm{X}$ was the shortest (Figs. $16 \mathrm{a}$ to $\mathrm{g})$. The total length, relative length, the centromeric position, the extent of Eu- and heterochromatin and number and position of chromomeres have been taken into account in depicting the pachytene chromosomes of the species under study. The absolute values concerning average length, relative length and arm-ratio of the chromosomes are presented in Table 1 specieswise. The chief diagnostic morphological characteristics of each of the ten pachytene chromosomes in the haploid complement are given below in ten 





different classes pointing out the similarities and differences amongst them. It may be noted, however, that for any given species the comparison is inter-class and for any given chromosome intra-class.

Table 2. Showing classification of chromosome I to $\mathrm{X}$ of seven species based on presence of chromomeres

\begin{tabular}{ll|c|c|c|c|c|c|c}
\hline & Species & $\begin{array}{c}\text { Chromo- } \\
\text { meres on } \\
\text { both arms }\end{array}$ & \multicolumn{2}{|c|}{$\begin{array}{c}\text { No. of chromomeres } \\
\text { on long arm }\end{array}$} & \multicolumn{2}{|c|}{$\begin{array}{c}\text { No. of chromomeres } \\
\text { on the short arm }\end{array}$} & No chromo- \\
meres
\end{tabular}

Chromosome I (mean length for different species range from $73.7 \mu$ to $81.1 \mu$ ). This longest chromosome of the complement is associated with the nucleolusthe nucleolus organizing region being situated on the short arm. Pycnotic regions are almost equally distributed on both the arms. However, a slight variation is noted with regard to chromomeric pattern in the different species (see Table 2, Figs. 16 a to g).

Chromosome II $(61.4 \mu$ to $67.5 \mu)$. The second longest of the complement, is asymmetrical in all the species except in $S$. bicolor in which it is symmetrical. Pycnotic regions are distributed more or less equally on both the arms in all the species except in $S$. mellitum where the short arm has a large pycnotic region than that on the long arm and in $S$. bicolor which has a shorter pycnotic region on the short arm as compared to that on the long arm. Variation is noted in the number of chromomeres on the two arms in the different species (Table 2 Figs. 16a to $\mathrm{g}$ ).

Figs. 1-13. 1-3, pachytene chromosomes $(n=10)$ of $S$. halepense, $S$. melaleucum, and $S$. technicum respectively. $\times 1600.4-7$, ears of parents and hybrids. Left-Female, MiddleHybrids, Right-Male. 4, S. nitens $\times$ S. caudatum. 5, S. nitens $\times S$. caffrorum. 6, S. vulgare $\times S$. verticilliforum. $7, S$. melaleucum $\times S$. vulgare. $8-9$, pachytene stages in the $F_{1}$ of $S$. verticilliflorum $\times S$. subglabrascens showing terminal as well interstitial nonpaired region. $(\uparrow) \times 1800$. 10, pachytene stage in the $F_{1}$ of $S$. nitens $\times S$. caudatum exhibiting terminal nonpairing regions $(\uparrow) \times 1800$. 11, pachytene chromosomes in $F_{1}$ of $S$. nitens $\times S$. caffrorum showing small terminal non-paired region and small duplication $\operatorname{Ioop}(\uparrow) . \times 1600.12$, pachytene stage in $F_{1}$ of $S$. vulgare $\times S$. verticilliflorum showing terminal deletions and unpaired regions $(\uparrow), \times 1800.13$, pachytene chromosomes in the $F_{1}$ of $S$. melaleucum $\times S$. vulgare showing normal pairing. $\times 1600$. 


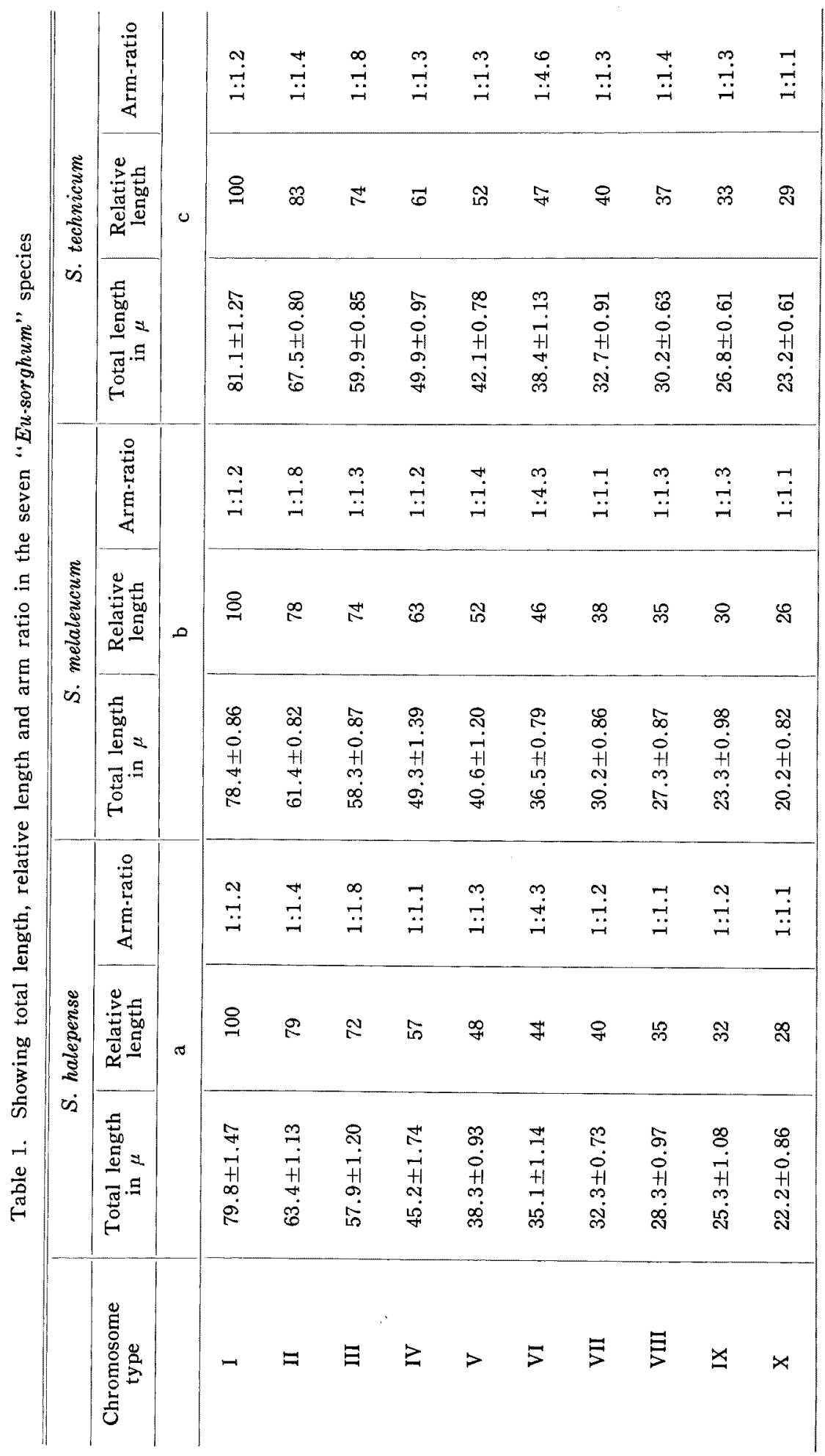




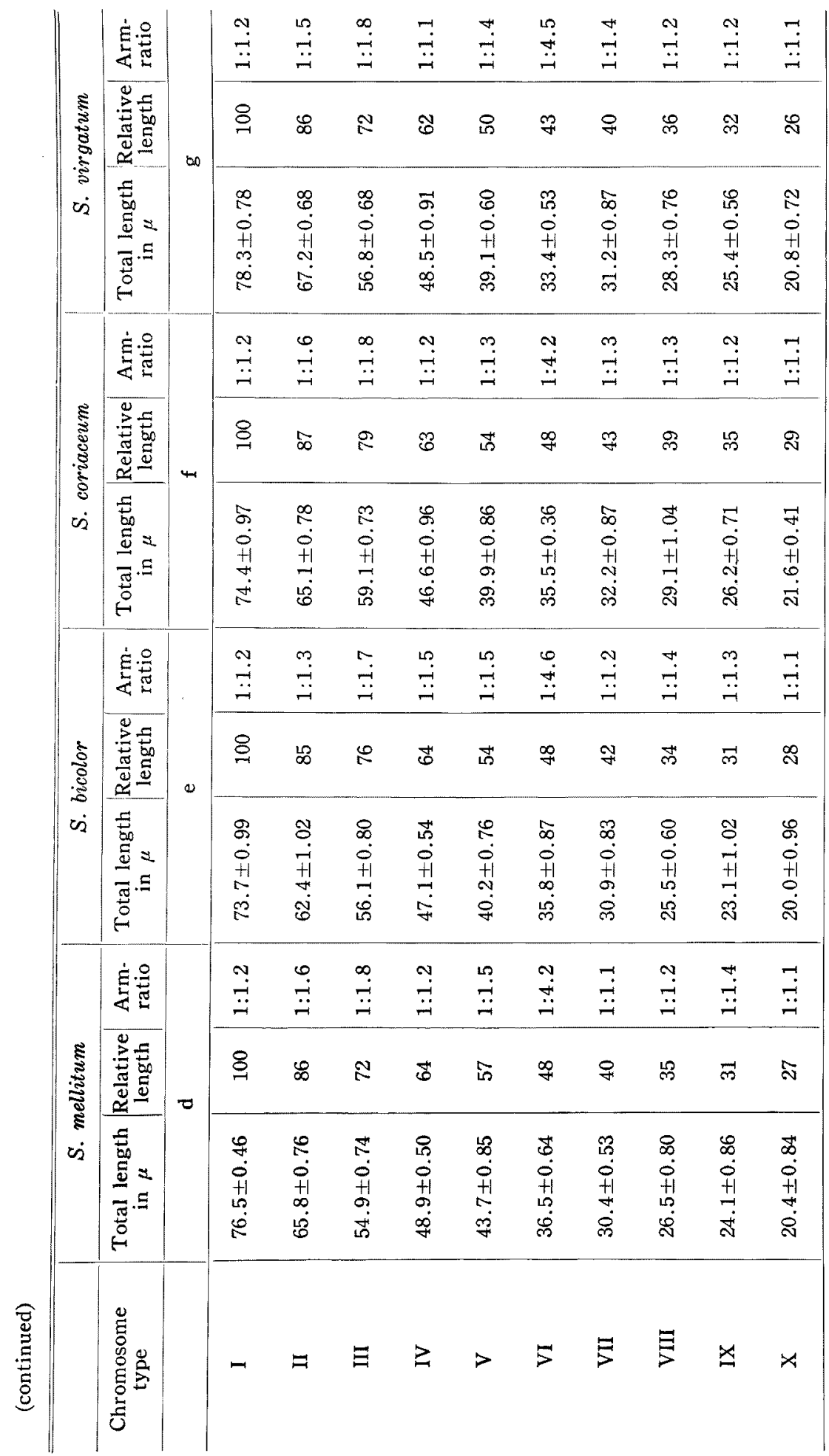


Chromosome III $(54.9 \mu$ to $59.9 \mu)$. Asymmetrical in all the species except in the case of $S$. melaleucum where it is nearly symmetrical. Pycnotic regions are distributed almost equally on both the arms except in the case of $S$. halepense and $S$. technicum where such regions are found to be larger on the short arm. The pattern of chromomeric variation on the two arms in these species is given in Table 2 (Figs. 16 a to g).

Chromosome IV $(45.2 \mu$ to $49.9 \mu)$. More or less symmetrical in all these species. Pycnotic regions are nearly equally distributed on both the

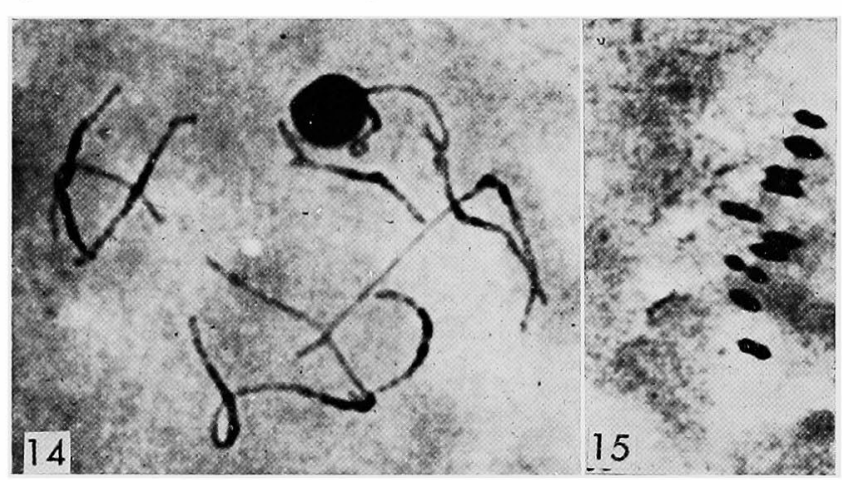

Figs. 14-15. 14, pachytene chromosomes in the $F_{1}$ of $S$. technicum $\times S$. virgatum showing normal pairing. $\times 1600$. 15 , metaphase I in the $\mathrm{F}_{1}$ of $S$. vulgare $\times S$. verticilliflorum showing 10 II $(\times 1600)$. arms except in the case of $S$. virgatum where the short arm has a larger pycnotic region as compared to that on the long arm. The variation noted as regards distribution of chromomeres on both the arms in these species may be seen in Table 2 (Figs. 16 a to $\mathrm{g}$ ).

Chromosome V $(38.3 \mu$ to $43.7 \mu$ ). This is more or less symmetrical in all these species. Pycnotic regions are distributed almost equally on both the arms except in the case of $S$. melaleucum where it is larger on the short arm than that on the long arm. The differences of the chromomeric pattern is given in Table 2 (Figs. 16 a to $\mathrm{g})$.

Chromosome VI $(33.4 \mu$ to $38.4 \mu)$. This is asymmetrical chromosome in the complement in each of the species. Except in S. technicum where the pycnotic region on the long arm is larger than that on the short arm, an almost equal distribution of pycnotic regions on both the arms is a characteristic feature of the remaining six species. The pycnotic region occupies about $3 / 4$ th of the entire length on the short arm. As regards chromomeric pattern, the details are given in Table 2 (Figs. 16 a to g).

Chromosome VII $(30.2 \mu$ to $32.7 \mu)$. This chromosome does not exhibit much variation in respect of relative length, arm-ratio etc. Pycnotic regions are distributed almost equally on both the arms except in S. technicum and $S$. bicolor where the pycnotic region is larger on the long arm as compared to that on the short arm. Whereas in $S$. virgatum it is larger on the short arm than that on the long arm. For variation in the chromomeric pattern refer Table 2 (Figs. 16 a to g).

Chromosome VIII $(25.5 \mu$ to $30.2 \mu)$. This chromosome is more or less symmetrical. Except in $S$. melaleucum where the pycnotic region is larger 
on the long arm than that on the short arm, the pycnotic region is distributed almost equally on both the arms. The differences amongst the species on the basis of chromomeric pattern in this chromosome are given in Table 2 (Figs. 16 a to $\mathrm{g}$ ).

Chromosome IX $(23.1 \mu$ to $26.8 \mu)$. This is a more or less symmetrical chromosome in the species under study. Except in S. halepense where two deeply staining regions are present on the short arm, proximal one being larger than the terminal, in all other species the deeply staining region is distributed almost equally on both arms. The differences between species in their chromomeric pattern are given in Table 2 (Figs. 16 a to g).

Chromosome $\mathrm{X}(20.4 \mu$ to $23.2 \mu)$. This symmetrical chromosome is strikingly uniform in all the seven species, being shortest in the complement.

Pycnotic region occupies comparatively larger part of this chromosome on both the arms than in all the other nine chromosomes in the complement. This chromosome is generally devoid of any
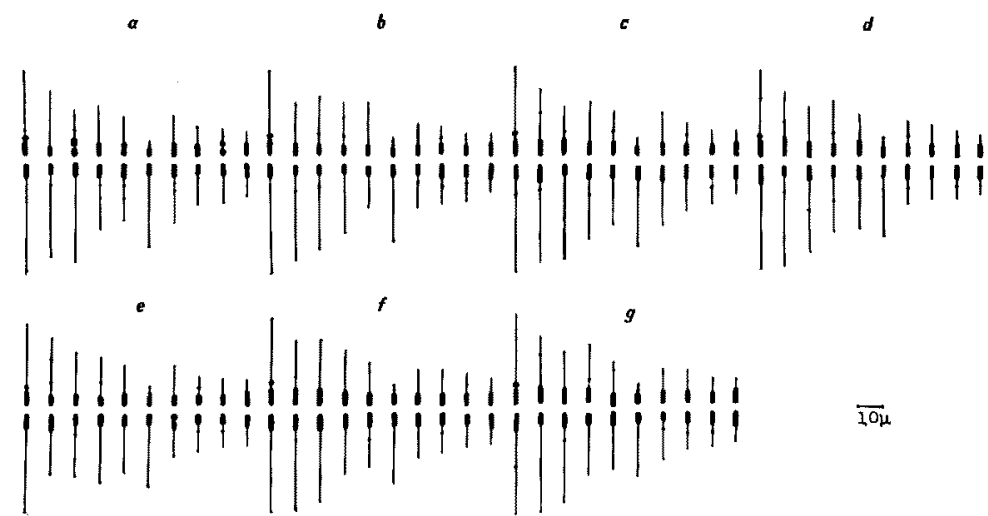

Fig. 16. a-g, showing idiograms. a, S. halepense. b, S. melaleucum. c, S. technicum, d, S. mellitum, e, S. bicolor, f, S. coriaceum and g, chromomere.

S. virgatum

All species under study show more or less similar staining behaviour of pachytene chromosomes. In each pachytene chromosome it was possible to observe a distinctly translucent centromere followed by deeply stained chromatic regions adjacent to it on either side. Pachytene pairing in all the species was found to be complete along the entire length and is apparently normal. Analysis of the pairing properties of the differentially stained regions indicated that synapsis starts from the proximal to the distal end. The separation of the split chromosomes starts from the distal to the proximal.

Ten bivalents were usually present both at diakinesis and M I. Chiasma frequency determined at these stages is presented in Table 3. The later stages of meiosis were also normal accompained by high percentage of stainable pollen and good seed set.

b) Species hybrids

The comparative morphological characteristics of the parents and the hybrids are given in Table 4 (Figs. 4 to 7 ). For characters such as tillering, 
Table 3. Showing chiasma frequency in the species hybrids

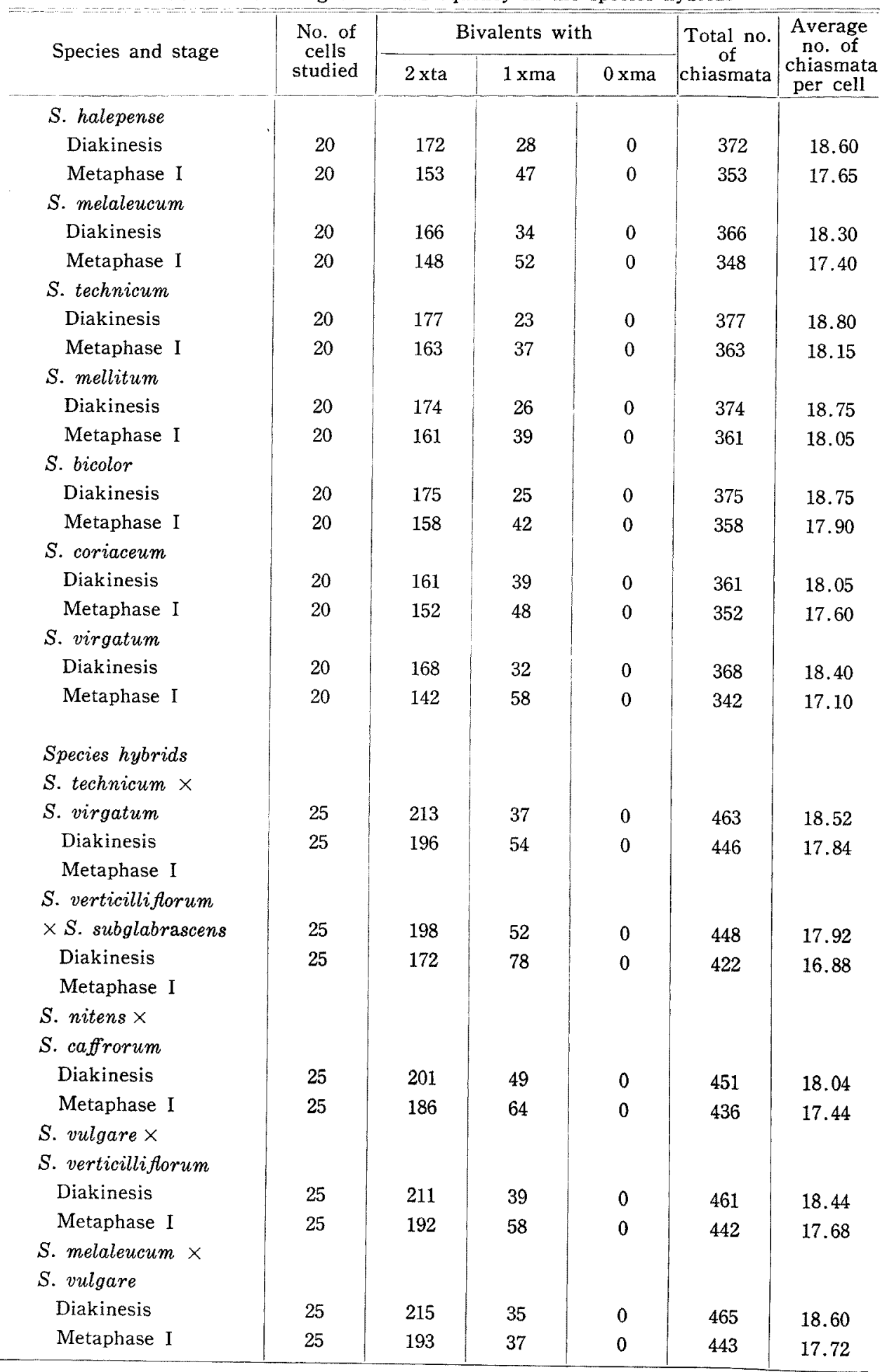




\begin{tabular}{|c|c|c|c|c|c|c|c|c|c|c|c|c|c|}
\hline 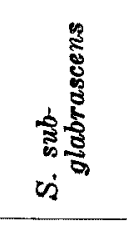 & 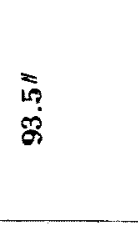 & $\stackrel{p}{\infty}$ & "ై & $\stackrel{\overline{\text { to }}}{\stackrel{\mathrm{s}}{ }}$ & 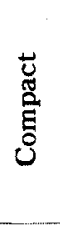 & 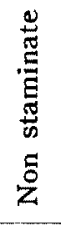 & $\begin{array}{l}\stackrel{0}{0} \\
\stackrel{0}{0} \\
0 \\
0\end{array}$ & 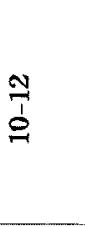 & $\stackrel{i}{2}$ & $\frac{\frac{\mathscr{U}}{\tilde{W}}}{\frac{3}{5}}$ & 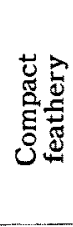 & 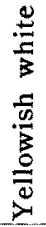 & క్ \\
\hline 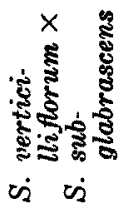 & 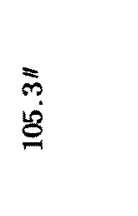 & $\frac{0}{b}$ & $\begin{array}{l}\stackrel{े}{1} \\
\stackrel{d}{*}\end{array}$ & \begin{tabular}{l}
$\overrightarrow{0}$ \\
\multirow{N}{*}{}
\end{tabular} & 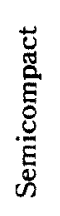 & 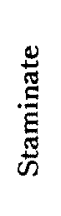 & $\frac{\stackrel{\infty}{E}}{\stackrel{0}{0}}$ & 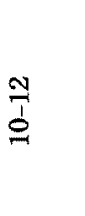 & 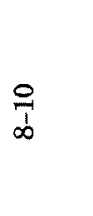 & 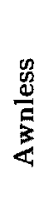 & 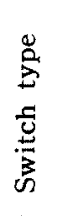 & $\frac{\vec{b}}{\bar{D}}$ & 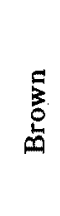 \\
\hline 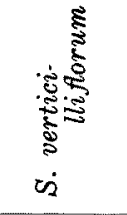 & $\begin{array}{l}\overline{0} \\
8\end{array}$ & $\begin{array}{l}9 \\
\infty\end{array}$ & $\begin{array}{l}\overline{10} \\
\dot{0} \\
\dot{0}\end{array}$ & $\stackrel{\Xi}{\circ}$ & 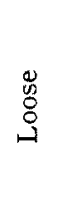 & 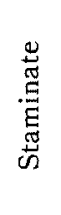 & 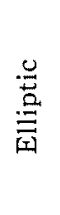 & )ิ & $\stackrel{R}{R}$ & 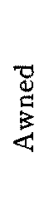 & 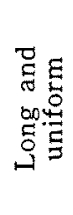 & $\frac{5}{0}$ & 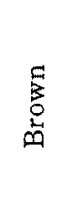 \\
\hline 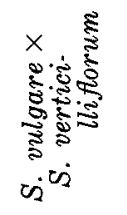 & : & d & $\begin{array}{l}\bar{m} \\
\text { s. } \\
\text { Is }\end{array}$ & $\begin{array}{l}\bar{O} \\
\dot{i}\end{array}$ & 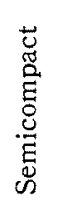 & 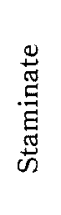 & $\begin{array}{l}\text { bo } \\
\stackrel{0}{0} \\
\stackrel{0}{0}\end{array}$ & 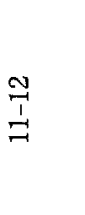 & $\stackrel{R}{2}$ & 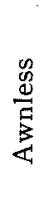 & 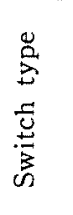 & & $\stackrel{\stackrel{D}{ت}}{\stackrel{D}{3}}$ \\
\hline 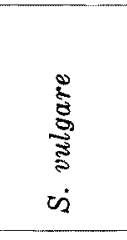 & 8 & $\frac{0}{1}$ & 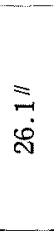 & $\begin{array}{l}\bar{m} \\
\bar{N}\end{array}$ & 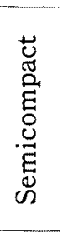 & 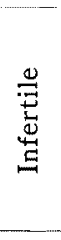 & $\begin{array}{l}\stackrel{Ð}{*} \\
\text { 今 } \\
0 \\
0\end{array}$ & $\begin{array}{l}\stackrel{1}{I} \\
\stackrel{1}{\leftrightarrows}\end{array}$ & $\stackrel{\$}{\infty}$ & $\frac{\stackrel{0}{0}}{\stackrel{0}{Z}}$ & 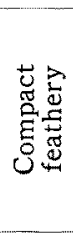 & $\begin{array}{l}\stackrel{0}{\leftrightarrows} \\
\frac{3}{3} \\
\frac{0}{3} \\
\frac{0}{3} \\
\frac{0}{0} \\
0-1\end{array}$ & 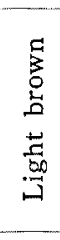 \\
\hline 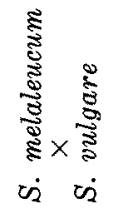 & $\begin{array}{l}\bar{\delta} \\
\dot{8}\end{array}$ & $\Phi$ & 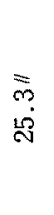 & $\overrightarrow{\vec{i}}$ & 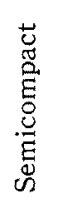 & 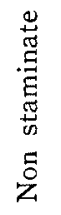 & 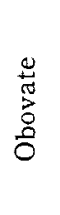 & $\stackrel{m}{\stackrel{M}{\leftrightarrows}}$ & 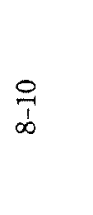 & 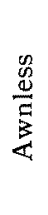 & 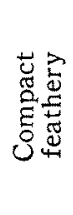 & $\frac{3}{\frac{3}{d}}$ & 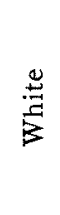 \\
\hline 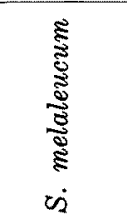 & $\begin{array}{l}\stackrel{\bar{N}}{\infty} \\
\ddot{\infty}\end{array}$ & $\begin{array}{l}a \\
\infty\end{array}$ & $\begin{array}{l}\bar{i} \\
\text { in }\end{array}$ & $\begin{array}{l}\bar{O} \\
\dot{N}\end{array}$ & 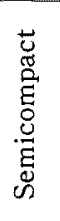 & 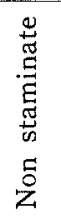 & $\begin{array}{l}0 \\
\stackrel{0}{5} \\
0 \\
0 \\
0\end{array}$ & 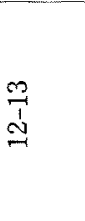 & $\frac{\circ}{1}$ & 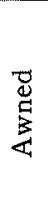 & 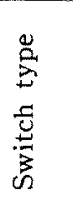 & 䓌 & $\stackrel{\stackrel{D}{:}}{\stackrel{乛}{5}}$ \\
\hline 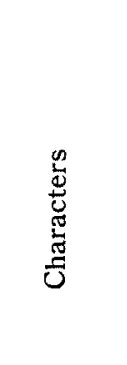 & 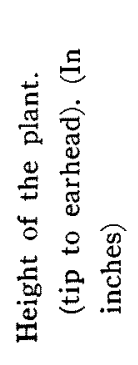 & 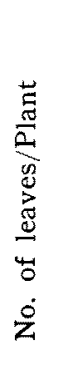 & 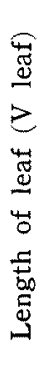 & 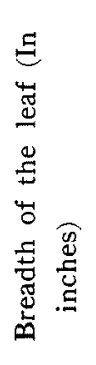 & 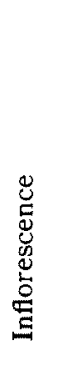 & 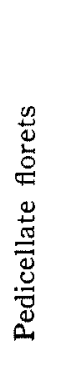 & 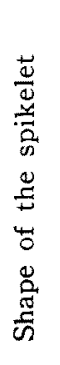 & 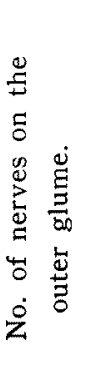 & 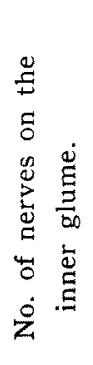 & 莺 & 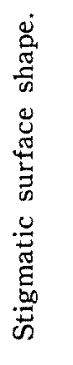 & 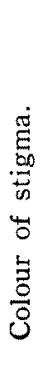 & 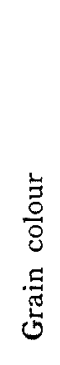 \\
\hline
\end{tabular}




\begin{tabular}{|c|c|c|c|c|c|c|c|c|c|c|}
\hline 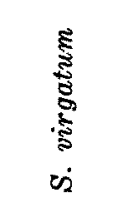 & $\underset{\infty}{\bar{j}}$ & $\begin{array}{ll}0 & 1 \\
\infty & \vdots\end{array}$ & స్ & 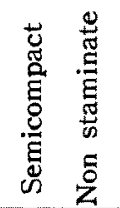 & & $\stackrel{\stackrel{N}{I}}{\underset{\exists}{\exists}}$ & $\frac{0}{3}$ & 总 & & 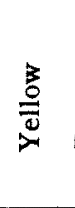 \\
\hline 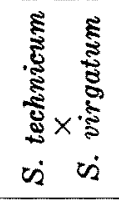 & 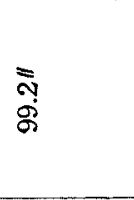 & $\begin{array}{l}7 \\
\overrightarrow{7} \\
0\end{array}$ & 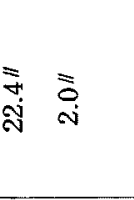 & 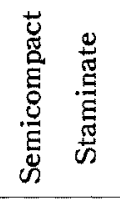 & $\begin{array}{l}\frac{0}{\pi} \\
\text { D. } \\
0 \\
0\end{array}$ & $\stackrel{\mathfrak{I}}{\stackrel{1}{二}}$ & $\stackrel{0}{\circ}$ & 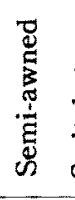 & 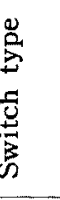 & \\
\hline 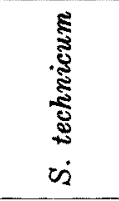 & సั่ & $\begin{array}{ll}\circ \\
\vdots \\
0\end{array}$ & $\begin{array}{l}\overline{0} \\
\stackrel{\infty}{\infty}\end{array}$ & 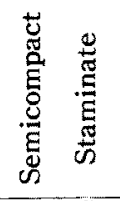 & 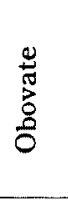 & $\stackrel{9}{I}$ & $\frac{9}{9}$ & 量 & 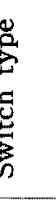 & $\stackrel{\stackrel{2}{3}}{3}$ \\
\hline 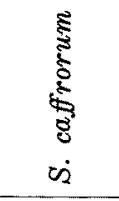 & $\begin{array}{l}\bar{c} \\
\infty \\
\infty \\
\infty\end{array}$ & $\begin{array}{ll}0 & \bar{c} \\
\infty & 0 \\
\infty & 0\end{array}$ & 佩 & 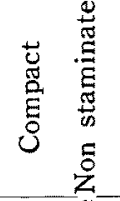 & 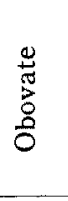 & 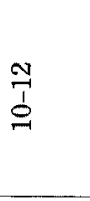 & $\underset{1}{\infty}$ & $\frac{\substack{0 \\
\frac{0}{4}}}{4}$ & 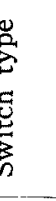 & $\stackrel{2}{3}$ \\
\hline 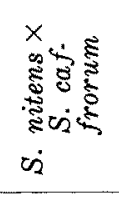 & 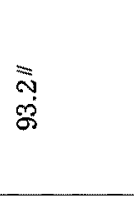 & 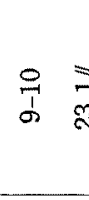 & 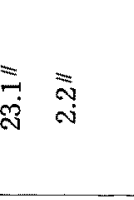 & 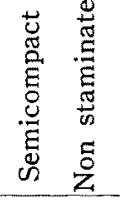 & $\begin{array}{l}\text { 总 } \\
\text { 范 } \\
\text { Oे }\end{array}$ & $\overrightarrow{\vec{b}}$ & 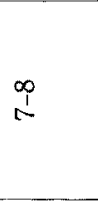 & 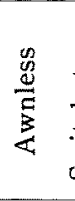 & 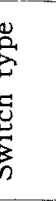 & 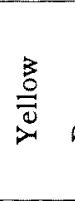 \\
\hline 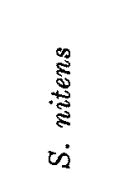 & 旁 & $\begin{array}{ll}\stackrel{0}{1} \\
\infty \\
\text { है }\end{array}$ & 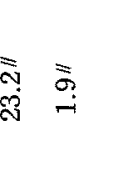 & 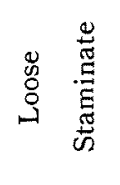 & 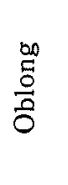 & 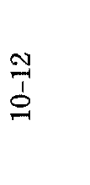 & i & $\frac{n}{0}$ & 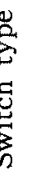 & 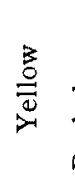 \\
\hline 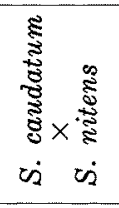 & ले & $\begin{array}{l}\circ \\
\bar{\alpha}\end{array}$ & 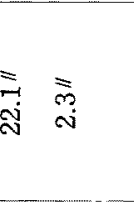 & 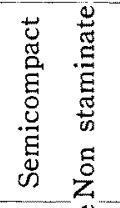 & 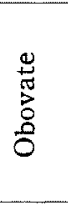 & $\overrightarrow{\bar{c}}$ & $i$ & : & 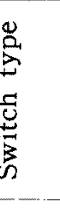 & 总 \\
\hline 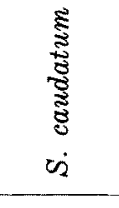 & $\begin{array}{l}\overline{0} \\
\dot{\infty} \\
\infty\end{array}$ & $\begin{array}{ll}0 & = \\
\infty & \\
\infty & \end{array}$ & 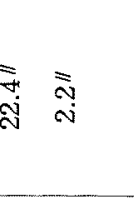 & 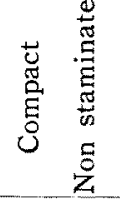 & 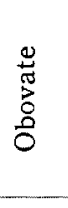 & $\stackrel{ㄱ}{\stackrel{\jmath}{g}}$ & $\begin{array}{l}\infty \\
\stackrel{1}{n}\end{array}$ & 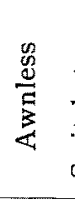 & 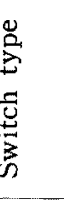 & $\stackrel{9}{:}$ \\
\hline 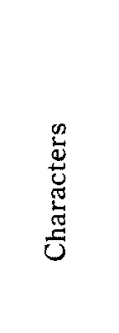 & 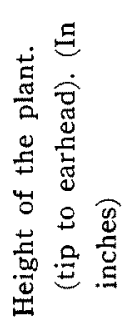 & 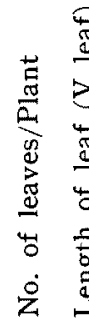 & 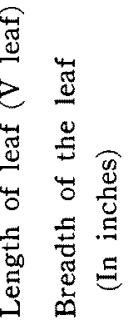 & 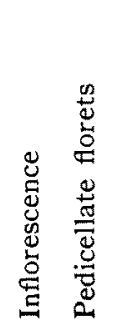 & 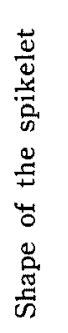 & 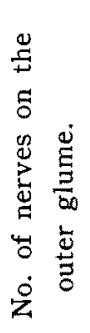 & 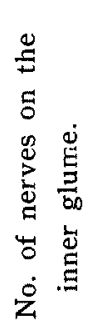 & 莺 & 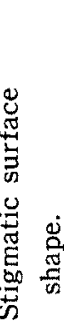 & 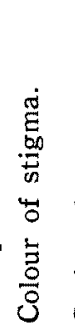 \\
\hline
\end{tabular}


panicle branching, floret shape, colour of the midrib, stigma and grains, the hybrids were intermediate. But in respect of characters such as plant height, leaf length and number of nodes the hybrids were usually heterotic. The awned condition was found to be recessive to awnlessness and the nonstaminate condition of pedicellate florets to staminate condition (see Table 4 for details).

Observations on the synapsis of the chromosomes at pachytene showed deviation from the normal meiosis noted in the parental species in some of the hybrids under study. In the hybrids, $S$. verticilliflorum $\times S$. subglabrascens, two bivalents showed non-paired regions, the unpaired regions being terminal in one and both terminal as well as interstitial in the other (Figs. 8 and 9). Again, in the case of two hybrids, S. nitens $\times S$. caudatum and $S$. nitens $\times S$. caffrorum, one to two bivalents respectively exhibited small terminal non-pairing regions (Figs. 10, 11). In the later hybrid, one bivalent also exhibited a small loop like structure indicating the presence of a small duplication (Fig. 11). In the hybrids, $S$. vulgare $\times S$, verticilliflorum, four bivalents showed large terminal non-paired regions as well as small terminal deletions (Fig. 12). In the same hybrid, one bivalent also exhibited interstitial unpaired region (Fig. 12). The pachytene pairing in the remaining two hybrids was complete for the entire length of the chromosomes and apparently normal (Figs. 13 to 14). It is interesting to note that inspite of the existence of such cytologically detectable though minute structural differences in some of the hybrids, the later stages of meiosis were normal in all the hybrids. $10 \mathrm{II}$ were observed both at diakinesis and MI (Fig. 15). The chiasma frequency determined at these stages is presented in Table 5. All the hybrids showed high percentage of stainable pollen and good seed set.

\section{Discussion}

It will be obvious from the results obtained in the present investigation and those already reported by Magoon et al. (1961) that the Sorghum material appears to be exceedingly suitable for pachytene analysis. In none of the species non-homologous pairing or stickiness was observed thereby removing this possible source of error. Further, centromeres are sharply definable in each of the pachyytene chromosomes in these species. However, conspicuous morphological land marks such as the presence of deeply staining knobs, were unfortunately not met with in the 20 chromosomes material studied and hence the identification of the individual chromosome was based on total length, relative length, centromeric position, amount and distribution of heterochromatin and number and position of chromomeres.

Considering all the available observations made so far on the morphology of the pachytene chromosomes in many crop plants, pachytene chromosomes may be broadly classified as differentiated and undifferentiated types (see review by Venkateswarlu 1962). The former category is characterized by 
proximal darkly staining region and distantly placed lightly staining region. The second type consists of chromosomes which take uniform stain or show chromomere size gradient. In this aspect the species studied in the present investigation and other Eu-sorghum species investigated by earlier workers (Venkateswaralu and Reddi 1956, Magoon and Shambulingappa 1960 and 1962 a and b, Magoon et al. 1961 a and b, Magoon and Ramanna 1961 a and b) may be classified under the differentiated type. It should be noted, however, that this type is not a characteristic of the entire genus since undifferentiated types of chromosomes have also been reported by some workers in certain species belonging to different sections. Nevertheless, the species of the section Eu-sorghum reported here and also studied previously appear to belong to the differentiated group.

The comparative study of pachytene chromosomes in the species under investigation brings out a good similarity in their general morphology. However, some differences are also recorded between them. It would be evident from the data (Tables 1 and 2, Fig. 16 a to g) that in general certain chromosomes do not exhibit much variation except for chromomeric pattern and minor variations in arm-ratios in a few cases. On the other hand, some other chromosomes show comparatively greater variations in their arm-ratio, pattern of pycnotic regions and distribution of chromomeres and thus might have undergone more structural alterations during the course of differentiation of these species. The length measurement between the longest and the shortest chromosome in the complement of these seven species show significant differences; the shortest chromosome (X chromosome) range from $20 \mu$ to $23.2 \mu$ and the longest chromosome (I chromosome) being $73.7 \mu$ to $81.1 \mu$ (Table 1). The proportion of the longest chromosome to the shortest in each of the species is little more than $1: 3$. The karyotype of each species also possesses at least one chromosome, usually VI, whose arm-ratio is more than $1: 4$. Following the classification proposed by Stebbins (1958), all the seven karyotypes studied here fall under ' $2 \mathrm{~b}$ ' type of asymmetry class. Several other Eu-sorghum species studied earlier (see Magoon and Shambulingappa 1960, Magoon et al. 1961 a and b, Magoon and Ramanna 1961 $a$ and $b$ ) could be classed under ' 2 b' type of asymmetry class.

The species under consideration also show interesting chromomeric pattern (Table 2, Fig. 16 a to g). Four distinct types can be recognized such as a) chromomeres on both the arms, b) chromomeres only on the long arm, c) chromomeres on the short arm, and d) no chromomeres at all on both the arms. It may be interesting to note that each species differ from the other with regard to chromomeric pattern in one or more chromomeres and thus this characteristic, in combination with relative length, arm-ratio etc. has proved to be of great help in characterizing the species. A comparison of the species based on the amount and distribution of heterochromatin is not attemped here since the measurement of relative lengths of the pycnotic 
areas could not be made accurately, as their demarcations were not very sharp. However, unequal distribution of pycnotic regions could be clearly observed in some instance, for example, II chromosome in $S$. mellitum, $S$. bicolor and $S$. virgatum and IV chromosome in $S$. virgatum. The data on the characteristics of the pachytene chromosomes being reported in the present study, though very informative is most rewarding when considered along with taxonomic criteria.

The cytological analysis of hybrids in recent years have been of great value in determining the homologies and consequently the probable relationships and origin of many species. A knowledge of cytogenetic affinities between cultivated species and their wild relatives has proved to be useful in utilizing the desirable germ plasm in the most effective manner for the improvement of the cultivated species through hybridization. The species of Sorghum belonging to the subsection Arundinacea show a striking similarity in both number and morphology of chromosomes (Magoon et al. 1961 a, Magoon and Ramanna 1961 a and b, Magoon 1961). Such studies have revealed the fact that majority of the Arundinancea Sorghum appear to be closely related fith one another. It must be emphasized, however, that structural differences (minute as well as patent), if any, may not be detectable by simply coniparing the karyotypes of different species. Hence analysis of chromosome pairing especially at pachytene in the hybrids is very essential not only to detect such structural changes but will also be helpful in understanding the cytogenetical mechanisms underlying species differentiation among the morphologically identified species in the genus Sorghum. This may be of immense value in planning hybridization programme.

It has been emphasized Stebbins (1947) and Magoon et al. (1962) that structural differences between the chromosomes could conceivally co-exist with a high degree of regular meiotic pairing if it is assumed that the chromosomes sets, concerned differ by a large number of small structural differences. Such 'cryptic' structural differences may not be able to offer much hindrance to pairing between chromosomes concerned and meiosis would appear to be apparently normal. However, they may be able to effectively prevent the free exchange of genes located within or very close to such regions and thus lay the foundation for the differentiation of the taxa concerned into different species. Consequently the breeder may not be able to recover the recombinant types for which he plans the crosses.

Sharma and Bhattacharjee (1957) reported marked differences in the somatic idiograms of several Eu-sorghum species studied by them and attribute species differences as arising out of gross structural changes. On the other hand, Magoon and Shambulingappa (1960), Magoon and Ramanna (1960 a and b) and Magoon et al. (1961 b) have demonstrated that the karyotypes of different species do not show much variation and therefore suggested that the species differentiation in the subsection Arundinacea of Eu-sorghums 
may be largely due to genic and cryptic or small structural changes. The study of species and their hybrids reported here confirm this view. Further, Sharma and Bhattacharjee (1957) have predicted highly irregular meiosis in the species hybrids of the subsection Arundinacea of Eu-sorghum on the basis of the marked karyotypic differences noted by them. On the contrary, remarkable regular meiosis, followed by normal pollen and seed fertility appears to characterize several species hybrids studied so far (Magoon and Shambulingappa $1962 \mathrm{a}$ and b). This has also been confirmed by other workers (see Kid 1956, Laubscher 1945, and Endrizzi 1957). These observations would lead one to suspect that species differentiation may be perhaps attributed largely to modifier complexes by which species differ. However, consistent reduction in the mean chiasma frequency at diakinesis and M I in several of the species hybrids reported earlier may suggest a significant role of small structural differences detectable only at pachytene stage. The presence of small structural differences have been recorded in some hybrids reported by Magoon and Shambulingappa (1962 a and b), and the presence of such small structural differences in a few hybrids in the present study provide again direct evidence for the latter view. It, therefore, becomes necessary to look for a sensitive tool to detect minor structural differences, if any, between the chromosomes in the species. The critical analysis of pachytene chromosomes for the pairing behaviour in the hybrids nucleus would appear to be the appropriate tool and is of immense value for such analysis.

Nature of chromosome pairing at pachytene has been studied in six species hybrids in the present study and out of them the following four hybrids $(S$. verticilliflorum $\times S$. glabrascens, $S$. nitens $\times S$. caudatum, $S$. nitens $\times S$. caffrorum and $S$. vulgare $\times S$. verticilliflorum) showed at pachytene stage cytologically detectable though minute structural differences such as terminal and interstitial non-pairing regions, differential segments, terminal deletions and small duplication loop (Fig. 11). Inspite of the existence of such structural differences, the later stages of meiosis were generally normal accompanied by high pollen stainability and good seed setting. The chiasma frequency also did not show any significant deviation from those in the parental species thereby indicating a marked extent of homology between chromosomes than would be expected to explain bivalents noted at $M$ I for which even a single chiasma between two chromosomes would suffice. All these factors again point out that the existing chromosomal structural differences are quite small and do not lead to abnormal behaviour of the hybrids either in respect of morphological characters or in the meiotic process. The remaining two hybrids showed complete pairing at pachytene and normal meiosis at later stages, followed by high percentage of stainable pollen and good seed setting. The chiasma frequency also did not show any significant deviation from the parental species. Apparently the differences! between the 
species are to be sought at the genic level. They might be of the nature of modifier complexes.

Stebbins (1938) found no correlation between the extent of structural differences present in the chromosomes of two Paeonia species and hybrid sterility. He observed, for example, that heterozygosity for many small inversions of Paeonia delavayi, $P$. anomala and $P$. tritermata, does not at all reduce chiasma frequency at $\mathrm{M} I$ in these species. In the hybrid, $P$. albiflora $\times P$. tenuifolia, where there was considerable reduction in chiasma frequency, a significant reduction in pollen fertility and particularly seed setting, showed the presence of only one inversion. Müntzing (1938) reported that interspecific sterility in Galeopsis tetrahit $\times G$. speciosa is, mainly, caused by structural differences in the chromosomes. A direct correlation between pollen sterility and the occurrence of univalents at meiosis in Galeopsis hybrids was also observed. From this it would appear as if the two phenomena, viz; appearance of univalents at M I and pollen sterility, must be regarded as different manifestation of the same cause, namely structural differences between the forms crossed. However, even in such an instance a certain parallelism between the extent of pollen sterility and degree of meiotic irregularities might be expected, if these phenomena are both proportional to the size and number of structural differences as was recorded by Garber and Gorsic (1956) in Collinsia, where the correlation is very weak or absent it must then be attributed to qualitative differences between the different chromosome segments. When certain hybrids exhibit normal meiosis and high sterility then there is reason to assume that the structural differences are small in quantity but important qualitatively. At the lower most level this would be termed as genic differences. In other $F_{1}$ combinations, on the other hand, meiosis may be upset by large segmental differences, comprising parts of the chromosomes which, however, are relatively harmless to the viability of the gametes.

In the genus Sorghum, while there appears to be a good correlation in some interspecific hybrids between the extent of structural differences present in the chromosomes and the percentage of pollen fertility found in them, no such correlation was observed in some other hybrids (Shambulingappa 1962 and Magoon and Shambulingappa (unpublished)). This apparently indicates qualitative differences in structural hybridity and hence it may be likely that the structural differences present in different species may differ both qualitatively as well as quantitatively. Since in most of the hybrids under consideration, no such correlation between the extent of structural difference present in the chromosomes and pollen fertility is noted, it probably indicates that the structural differences observed in these hybrids are probably not as important qualitatively to affect the viability of gametes.

Interspecific hybrids have been successfully produced and investigated to provide information of cytogenetic and phylogenetic value of possible use in 
the improvement of Sorghum by hybridization. The results obtained in the present investigation and also reported earlier (Magoon and Shambulingappa $1962 \mathrm{a}, \mathrm{b}$ and c) and some unpublished work (Shambulingappa 1962) do suggest the possibility of transferring valuable germ plasm from both wild as well as cultivated species into the commercial variety since a majority of the present species of Eu-sorghum do not appear to be separated by any serious barrier to gene-exchange.

Considering the internal mechanisms responsible for speciation in this group of plants, it appears likely that gene mutations or gene substitution, 'cryptic' structural differences, small, though cytologically detectable, polyploidy and probably cytoplasmic differentiation seem to have played a signifcant role. The part played by the gross structural changes, if any, cannot be very significant. The striking fact, that emerges from a consideration of the crossability relationship of Eu-sorghum is that while most of the internal isolating mechanisms mentioned above are present, barriers to crossability between the species appear to be rather feebly developed. This becomes more apparent when we compare the situation prevailing in the section Para-sorghum of this genus, where they appear to be very strongly developed. From the point of view of the systematic taxonomist whether species distinctions are attributed to multiple gene substitution or to 'cryptic' or small structural differences is only of academic significance but for a geneticist it is an issue of prime importance. For, upon it depends the degree to which a breeder can hope to transfer a character of potential economic value from one species to another. If species differences are mainly due to multiple gene substitution, the problem is chiefly a matter of growing progenies large enough to secure the required combination. Whereas the success with which a breeder can expect to transfer valuable characters from one species to another will be inversely proportional to the frequency of 'cryptic' or small structural differences in his breeding material since the genes within these regions would tend to be passed on to the progeny enbloc (Magoon et al. 1962).

\section{Summary}

The morphological characters of nine species and six $F_{1}$ hybrids were compared. It was found that with respect to characters such as tillering, panicle branching, floret shape, colour of the midrib, stigma and grains, the hybrids were intermediate but for characters such as plant height, leaf length and number of nodes, the hybrids were usually heterotic. The awned condition was found to be recessive to awnlessness and the non-staminate condition of pedicellate florets to staminate condition.

Critical analysis of chromosomes at pachytene and later stages of microsporogenesis in the seven Eu-sorghum species and six $F_{1}$ hybrids were made. Pachytene chromosomes are depicted on the basis of total length, 
relative length, arm-ratio, amount and distribution of heterochromatin and number and position of chromomeres. The characteristics of each of the ten chromosomes in the haploid complement are described in ten different classes bringing out the similarities and differences among these seven species. Studies on the pairing properties of the differentially stained regions show that synapsis starts from the proximal to the distal and separation of the split chromosomes starts from the distal to the proximal.

Pachytene analysis in the four $F_{1}$ hybrids revealed the presence of some minute, though cytologically detectable, structural differences such as terminal as well as interstitial non-paired regions, small duplication and small terminl deletions and differential segments between the parental species. Pachytene pairing was found to be complete and apparently normal in the remaining two hybrids. Inspite of the existence of these meiotic irregularities in some hybrids, high percentage of stainable pollen and good seed setting were recorded in all the hybrids under consideration.

The distribution of chiasmata at various stages was studied in both species and species hybrids and the average chiasma frequency did not show any significant deviation from those in the parental species. It is suggested that the existing chromosomal differences are quite small and do not lead to abnormal behaviour of the hybrids either in respect of morphological characters or in the meiotic process in the $F_{i}$ generation.

Cytogenetical mechanisms underlying species differentiation in the genus are discussed.

\section{Acknowledgements}

The authors are grateful to Dr. B. P. Pal and Dr. M. S. Swaminathan for their keen interest and constant encouragement. Co-operation from the "Rockefeller Foundation", I.A.R.I., New Delhi, is also hereby acknowledged.

\section{References}

Barton, D. W. 1950. Pachytene morphology of the tomato chromosome complement. Amer. J. Bot. $37: 639-43$

Endrizzi, J. E. 1957. Cytological studies of some species and hybrids in Eu-sorghum. Bot. Gaz. 119: 1-10.

Garbar, E. D. and Gorsic, J. 1956. The genus Collinsia II. Interspecific hybrids involving C. heterophylla, C. coneolor and C. sparciflora. Bot. Gaz. 118: 73-77.

Gottschalk, W. 1954. Die Chromosomen Struktur der Solanaceen unter Berücksichtigung phylogenetischer Fragestellungen. Chromosoma 6: 539-626.

Huziwara, Y. 1956. Karyotype analysis in some genera of Compositea. I. Karyotype of Japanese Eupatorium. Cytologia 21: 114-123.

Kid, H. J. 1956. The morphology of the panicle in the cultivated Sorghums. Ph. D. dissertation. Washington Univ. St. Louis. (original not seen)

Laubscher, F. X. 1945. A genetic study of Sorghum relationship. Dept. Agric. (Union of South Africa). Sci. Bull. 242.

Magoon, M. L. 1961. Genetical and cytogenetical studies in the genus Sorghum. Proc. 
2nd. All. 1nd. Millet. Conf., Kanpur.

-, Cooper, D. C. and Hougas, R. W. 1958. Cytogenetic studies of some diploid Solanums, section Tuberarium. Amer. J. Bot. 45: 207-221.

-, Ramanujam, S. and Cooper, D. C. 1962. Cytogenetical studies in relation to the origin and differentiation of species in the genus Solanum L. Caryologia 15: 151-252.

- and Ramanna, M. S. 1961-a. Comparative karyomorphology of Eu-sorghums. Caryologia $14(3)$ : $391-407$.

- and - 1961-b. Cytology of some "Eu-sorghums". Genet. Iber. XIII 129-144.

- and Shambulingappa, K. G. 1960. Karyomorphological studies in Sorghum ankolib var. ankolib red, a Eu-sorghum. Indian J. Genet. 20: 166-177.

-- and - 1961. Karyamorphology in Sorghum propinquum and its bearing on the origin of 40-chromosome Sorghums. Chromosoma 12: 460-465.

- - and Ramanna, M. S. 1961-a. A polyhaploid plant in Sorghum halepense. Pers. Current Sci. 30: $347-48$.

-, - and -, 1961-b. Chromosomes morphology and meosis in some Eu-sorghums. Cytologia $26: 236-52$.

- and - 1962-a. Cytological and morphological studies on some interspecific hybrids in Eu-sorghum. Zeitschrift für Vererbungslehre 93: 14-24.

- and - 1962-b. Cytomorphological studies of some interspecific hybrids in the genus Sorghum. Der Züchter. 32: 317-324.

- and - 1962-c. A polyhaploid plant of S. almum. Genetica Polonica. 3: 301-306.

Müntzing, A. 1938. Sterility and chromosome pairing in intra-specific Galeopsis hybrids. Hereditas 24: 117-188.

Shambulingappa, K. G. 1962. Cytogenetical studies and nature of speciation in the genus Sorghum. Ph. D. dissertation I.A.R.I., New Delhi.

Sharma, A. K. and (Miss) Dipiti Bhattarcharjee 1957. Chromosome studies in Sorghum I. Cytologia 22: 287-311.

Stebbins, G. L. 1938. Cytogenetic studies in Paeonia II The cytology of diploid species and hybrids. Bot. Rev. 11: 463-86.

- 1947. Types of polyploids: their classification and significance. Adv. Rev. 11: 46329.

- 1958. Longivity, habitat and release of variablity in the higher plants. C.S.H.S. Quant. Biol. 23: 365-78.

Swaminathan, M. S., Magoon, M. L. and Mehra, K. L. 1954. A simple propiono-carmine PMC smear method for plants with small chromosomes. Indian J. Genet. 14: 8788.

Venkateswaralu, J. 1962. Some aspects of the study of pachytene chromosomes. 49 th Indian Sci. Congr. 1-20.

- and Reddi, R. 1956. Morphology of pachytene chromosomes and meiosis in Sorghum subglabrascens, a Eu-sorghum. J. Indian Bot. Soc. 35: 344-56. 